\title{
Les dimensions de la santé des jeunes
}

\section{Jean Martin}

Dr méd., membre de la rédaction

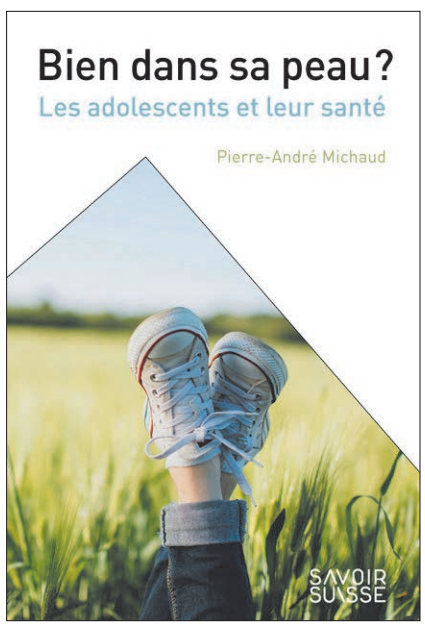

Pierre-André Michaud

Bien dans sa peau?

Les adolescents et leur santé

Lausanne: Presses polytechniques et universitaires romandes («Savoir suisse»), 2018.

160 pages. CHF 17.50 .

ISBN: 978-2-88915-270-4

Le professeur P.-A. Michaud est bien connu dans la vie médicale et universitaire suisse - et à l'étranger (il a notamment assumé des mandats pour l'OMS et l'UNICEF). Après avoir été médecin de famille en cabinet, il a été depuis 1998 le premier responsable de l'Unité multidisciplinaire de santé des adolescents au CHUV de Lausanne, puis vice-doyen de la faculté. A élaboré récemment d'importants rapports sur la formation médicale.

Ce livre a onze chapitres. Quelques titres: chap. 3: La puberté, une exception dans le monde animal; chap. 5: La santé mentale et les dérives psychopathologiques; chap. 6: Faire face à une affection chronique ou un handicap; chap. 7: Internet et les médias sociaux: trouver de bonnes réponses; chap. 9: Qu'est-ce qu'un usage nocif de drogue? Chacun est introduit par une vignette clinique.

On y trouve des mises au point sur les données physiologiques (de la puberté en particulier, de son évolution au cours du temps, de ses variations - elle peut être influencée par des perturbateurs endocriniens), sur le développement normal ou problématique du jeune, ce qui promeut ou au contraire peut altérer sa santé. Avec des données épidémiologiques et sociologiques, historiques aussi.

Des faits de base. «On ne saurait taire l'importance tenue dans un grand nombre de sociétés par les rites de passage, dont la fonction structurante est de donner un sens au monde, à la vie et à la mort. Mais dans la société actuelle globalisée, les adolescents ne bénéficient plus de tels rituels et doivent forger eux-mêmes leur système de valeurs.» "La nouvelle manière d'accéder au statut d'adulte doit beaucoup à des contextes radicalement nouveaux: absence relative de repères, multiculturalisme, ouverture au monde et accès instantané à l'information.» A cela s'ajoute «le décalage générationnel produit par les nouvelles technologies».

«Il faut souligner que ces 20 dernières années ont vu les taux de décès par mort violente diminuer en Suisse. Cela est lié à des mesures éducatives et sanitaires, mais aussi environnementales, comme l'amélioration des conditions de circulation, les limitations de vitesse, le port obligatoire du casque - et de la ceinture, la limitation de l'accès aux boissons alcoolisées ou l'accompagnement des jeunes consommant des drogues.»

Et: ne pas oublier que, «si l'adolescence en tant que telle est un phénomène nouveau dans l'évolution des sociétés, le fossé entre les générations lui a toujours existé»!

Comment juger des consommations? «Pour faire court, dire clairement - comme de fait l'affirme la loi que la consommation de toute substance psychoactive avant l'âge de 16 ans est potentiellement nocive pour la santé.» "Le cannabis agit comme un détonateur sur le cerveau d'un individu de 12-13 ans alors qu'une consommation occasionnelle en fin d'adolescence n'est pas grevée de risques.» "Toute consommation, surtout avant 17 ou 18 ans, doit être prise comme une activité symptomatique dont il importe de décoder, avec le jeune sujet, la signification pour lui.»

«Ce ne sont pas tant la nature du produit et les doses que les motifs et circonstances personnelles et environnementales qui permettront de définir la frontière entre un usage récréatif et un usage problématique.» Internet et médias sociaux: «Il est raisonnable d'adopter dans ce domaine une attitude d'ouverture critique, 
sans tomber dans la diabolisation ni dans une naïveté faite de confiance excessive ou d'absence de contrôle.» Des recommandations sont données sur l'usage des écrans à différents âges.

Sexualité, un des grands thèmes. "Beaucoup d'adultes tendent à se fixer sur les aspects physiques de la sexualité et abordent cette thématique comme un problème, alors que la vision qu'en ont les adolescents est beaucoup plus large et positive.» «En dépit des progrès réalisés ces dernières décennies, les stéréotypes de genre subsistent et les modèles féminins et masculins imprègnent les processus identitaires.»

Une partie non négligeable des adolescents (près de $30 \%$ ) déclare avoir été soumise à une forme de cyberharcèlement. «Il est parfois difficile pour les jeunes de définir eux-mêmes dans quelle mesure ils ont été victimes de violence ou non [...] Ils éprouvent parfois de la difficulté à savoir si la relation sexuelle était consensuelle ou non.»

«Depuis des années, la Suisse connaît l'un des taux les plus faibles de grossesses [chez les ados] et d'interruptions de grossesse du monde. Cette situation réjouissante est liée à la conjugaison de plusieurs facteurs: activités d'éducation sexuelle généralisées, du moins en Suisse romande, centres de planification familiale accessibles, vigoureuses campagnes sida; discours public marqué du sceau du pragmatisme, avec un cadre légal fixant la majorité sexuelle à 16 ans.»

«En dépit de la situation favorable que connaît la Suisse romande [...], il est important d'insister sur le fait que certaines mouvances politiques ou religieuses tendent régulièrement à remettre en question la pertinence des mesures de prévention.»

Pratique médicale et soignante. «Beaucoup de médecins tendent à sous-estimer la compétence (capacité de discernement) des adolescents. Les soignants doivent réserver à ceux-ci un espace de confidentialité au sein duquel ils puissent s'exprimer en toute liberté, sans pression." "Les médecins devraient apprendre à impliquer plus systématiquement leurs jeunes patients dans l'élaboration de leur traitement ou de mesures de prévention.»

NB: «Les adolescents vivant une maladie chronique ou un handicap restent ... des adolescents. Ils ne sont pas des diabétiques ou des asthmatiques, ils sont des adolescents souffrant de diabète ou d'asthme! [...] On comprend tout l'intérêt d'un accompagnement interdisciplinaire. Les médecins d'adultes ne sont souvent pas bien préparés à gérer les enjeux du suivi holistique d'un ado.» A propos de la vie familiale: "On mésestime parfois l'impact d'une affection chronique sur la fratrie; d'où l'importance d'encourager les parents à s'occuper aussi des frères et sœurs et de leurs besoins d'attention.»
La prévention, encore insuffisamment mise en œuvre. «Dans un pays à idéologie libérale, il est difficile de faire promulguer des directives qui permettraient d'éviter que les jeunes soient pris en otage par les grandes compagnies de distributions et d'alimentation.»

«La prévention passe avant tout par des mesures environnementales et structurelles plutôt qu'uniquement par des incitations éducatives. Si l'usage de tabac a diminué, c'est surtout en raison de l'évolution des normes (restriction des endroits où consommer), ainsi que de l'augmentation du prix [...] Les mesures interactives, incitant les adolescents à questionner leurs consommations, se sont montrées nettement plus efficaces que la simple information.»

"On devrait développer encore plus des approches structurelles comme un climat scolaire fait d'attention mutuelle, de respect des règles, de participation active des élèves.» "Les adolescents peuvent être amenés à jouer un rôle actif dans la prévention de divers comportements: par exemple des écoles ou des communautés forment des jeunes à l'identification de camarades en difficulté et à l'accompagnement de ceux-ci vers des services de soins.»

En guise de conclusion. Dans l'ensemble, les adolescents dans notre pays jouissent d'une bonne santé tant physique que psychosociale. De plus, la situation dans bien des domaines s'est améliorée récemment, que ce soit du point de vue de la violence ou de l'usage de drogue, par exemple.

«Donner la parole aux adolescents, c'est prendre en compte leur avis et accepter d'étudier toute proposition, si farfelue soit-elle. Par ailleurs, une participation effective ne peut avoir lieu qu'au prix de l'acquisition des 'compétences transversales', qui leur permettent de s'exprimer en présence d'adultes et de connaître leurs droits.»

«Il est difficile d'imaginer la situation d'ici 10 à 20 ans: disparition de l'écriture cursive? Quelle place occupera l'intelligence artificielle? Quelles seront les conséquences d'un 'multitasking' qui se généralise?»

Et un rien de politique de santé! «Il reste à souhaiter que notre société donne aux adolescents la place qui leur est due et que la part prise par un nombre grandissant de personnes âgées n'obère pas l'avenir de ces jeunes et ne monopolise pas les débats politiques.» Y réfléchir.

Bien dans sa peau? est, selon la manière de la collection "Savoir suisse», un ouvrage substantiel tout en étant concis, bien structuré, que tous ceux qui s'intéressent à la composante particulière de notre communauté, surprenante, voire énigmatique, que sont les adolescents liront avec grand intérêt. 